\title{
Newborns of COVID-19 mothers: short-term outcomes of colocating and breastfeeding from the pandemic's epicenter
}

\author{
Uday P. Patil $\mathbb{1}^{1,2} \cdot$ Sheela Maru ${ }^{2,3,4} \cdot$ Parvathy Krishnan ${ }^{1,2} \cdot$ Rachel Carroll-Bennett $^{2,4} \cdot$ Joselito Sanchez $^{1,2}$. \\ Lawrence Noble $\mathbb{i}^{1,2} \cdot$ Randi Wasserman ${ }^{1,2}$
}

Received: 18 May 2020 / Revised: 26 June 2020 / Accepted: 22 July 2020 / Published online: 10 August 2020

(c) Springer Nature America, Inc. 2020

\section{Introduction}

The United States of America has been severely affected by the COVID-19 pandemic during the spring of 2020 [1]. The largely immigrant and densely populated neighborhoods of Queens, NY, served by a large public hospital, Elmhurst Hospital Center (NYC H+H/Elmhurst), have emerged as one of the hardest-hit areas in the country [2]. Newborns are at high risk of acquiring severe acute respiratory syndrome coronavirus 2 (SARS-CoV-2) from their infected mothers who delivered during this period; however, data remains limited [3]. We aim to describe the unique experience from our Baby Friendly hospital at the epicenter of the COVID19 pandemic.

\section{Methods}

In this retrospective cross-sectional study, we identified live births to women who were tested for SARS-CoV-2 from March 19 to April 22, 2020 at NYC H+H/Elmhurst. Data regarding demographic and clinical characteristics, SARSCoV-2 test results, symptoms and signs of COVID-19, colocation (rooming-in), breastfeeding, and newborn follow-up were obtained from review of electronic medical

Uday P. Patil

uday.patil@mssm.edu

1 Department of Pediatrics, Icahn School of Medicine at Mount Sinai, NY, USA

2 New York City Health+Hospitals/Elmhurst, Elmhurst, NY, USA

3 Department of Health System Design and Global Health, Icahn School of Medicine at Mount Sinai, New York, NY, USA

4 Department of Obstetrics, Gynecology and Reproductive Science, Icahn School of Medicine at Mount Sinai, New York, NY, USA records. Descriptive statistics on the newborns of positive mothers along with maternal data is presented as overall results and percentages. BioReference laboratories and our hospital laboratory (Cepheid Xpert Xpress) were used for SARS-CoV-2 reverse transcriptase polymerase chain reaction (PCR) test. This study was approved under exempt status by the Institutional Review Board of Icahn School of Medicine at Mount Sinai (IRB number 20-03424), local ethics committee, and the office of research administration.

\section{Results}

There were 118 live births to women who were tested for SARS-CoV-2 infection. Of these, 45 (38\%) newborns were born to positive mothers and $30(67 \%)$ births were vaginal. The majority of positive mothers, 27 (60\%), were asymptomatic. Five $(11 \%)$ well newborns were placed in isolation room as a part of our initial practice early in the pandemic. Seven $(16 \%)$ newborns were admitted to the neonatal intensive care unit (NICU) after birth due to prematurity or suspected sepsis. A total of $33(73 \%)$ newborns colocated with their mothers and 31 of the $33(94 \%)$ were breastfed with the initiation of breastfeeding within $1 \mathrm{~h}$ of birth. All the newborns were tested for SARS-CoV-2 using a combined oropharyngeal and nasopharyngeal swab after birth following their first bath due to the long turnaround time of the test results. Testing from other sites such as rectal swabs was not performed. A total of $33(73 \%)$ newborns tested negative and $3(6.6 \%)$ tested positive (Fig. 1). Those three newborns were monitored in the NICU until two consecutive tests obtained at least $24 \mathrm{~h}$ apart were negative and they remained asymptomatic, thus suggesting transient colonization. None of the 45 newborns needed NICU admission for COVID-19-related symptoms. The average age at discharge was $2.7( \pm 2.3)$ days of life. Upon discharge, there was a $91 \%$ adherence (39 out of 43 discharged) to the initial in-person newborn visit. There was 
$100 \%$ adherence to the televisits within 2 weeks post discharge. On these visits, none of the newborns were reported to have any symptoms consistent with COVID-19 and none had emergency department visits or hospital admissions related to COVID-19. Demographic and clinical data is summarized in Table 1.

\section{Discussion}

In our experience of caring for newborns at a center with high incidence of maternal SARS-CoV-2, newborns did not show signs of infection after birth. Moreover, we report no shortterm adverse neonatal outcomes with skin-to-skin care, rooming-in, or breastfeeding in infants of SARS-CoV-2 positive mothers in our population. In contrast to early reports from China, majority of our newborns were born via vaginal delivery [4]. And although it has been shown that SARS$\mathrm{CoV}-2$ can be detected in the stool samples of mothers, routine testing in our cohort of newborns did not show a high incidence of positive PCR results [5]. A possible explanation for both of these observations could be the high number of asymptomatic or mildly symptomatic mothers in our study.

During the early period of the pandemic, our center tested only symptomatic pregnant women for SARS-CoV-2 and newborns of infected mothers were placed in isolation rooms based on the available literature at that time [6]. However, due to the dramatic surge in the number of SARS-CoV-2 positive mothers delivering at our center after initiation of universal screening of all pregnant women admitted for delivery, the limited isolation spaces for the newborns, early postpartum discharges, the crowded
Fig. 1 SARS-CoV-2 test results and clinical characteristics of the newborns and their mothers.

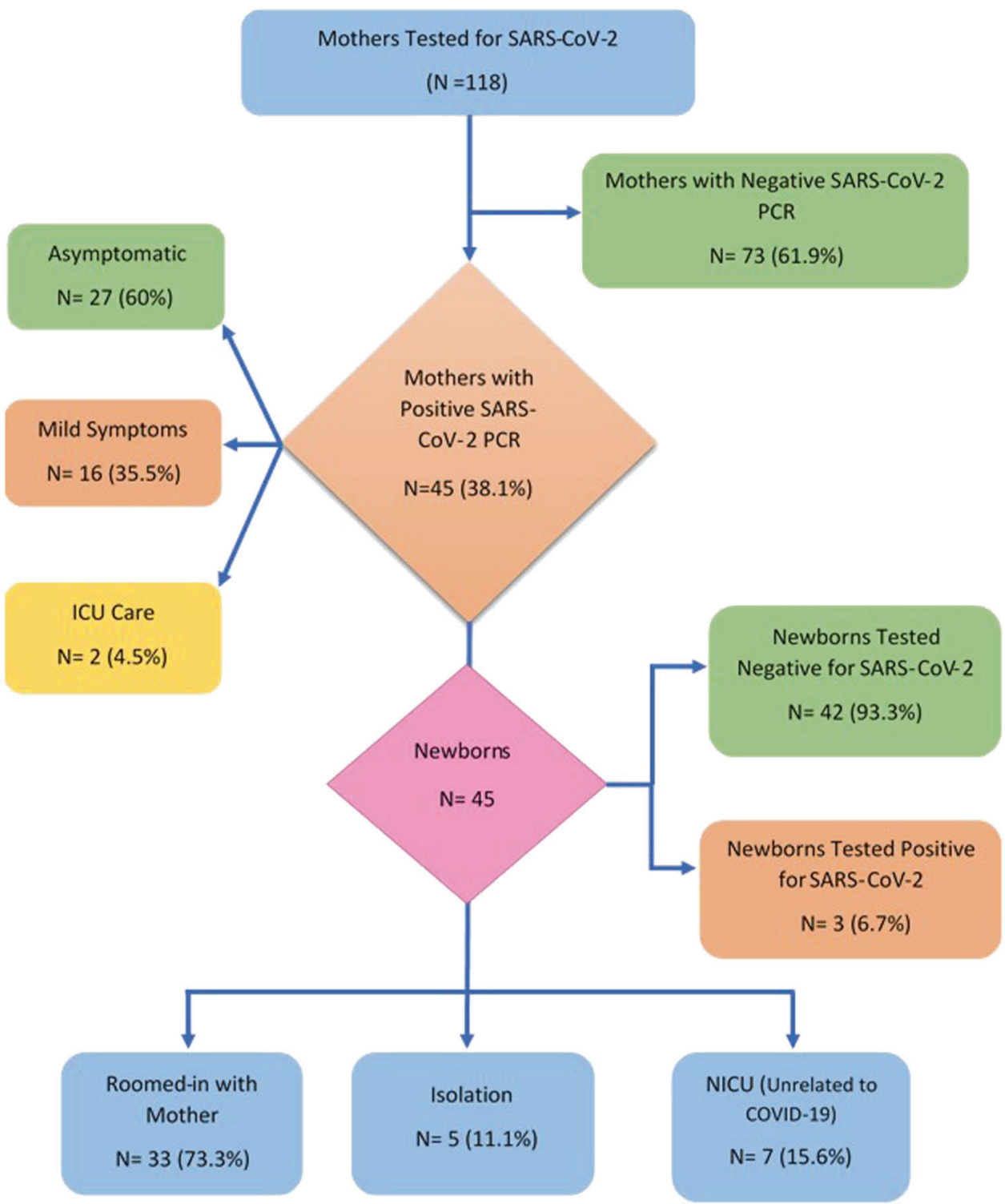


housing conditions in our community and the desire to promote breastfeeding, we utilized shared decision-making and offered rooming-in of mothers with their newborns [7]. Mothers were encouraged to provide skin-to-skin care and breastfeeding while rooming-in, following appropriate isolation precautions based on the CDC guidance [8].

Table 1 Demographic and clinical characteristics of newborns of SARS-CoV-2 positive mothers.

\begin{tabular}{|c|c|}
\hline Characteristics & $\begin{array}{l}\text { Patients/Value } \\
(N=45)\end{array}$ \\
\hline \multicolumn{2}{|l|}{ Maternal characteristics } \\
\hline Age, median (IQR), years & $30(27.5-35.5)$ \\
\hline Vaginal delivery, no. (\%) & $30(66.7)$ \\
\hline Cesarean section, no. (\%) & $15(33.3)$ \\
\hline Length of stay, median (IQR), days & $3(2-3)$ \\
\hline Asymptomatic, no. (\%) & $27(60)$ \\
\hline Symptomatic, no. (\%) & $18(40)$ \\
\hline \multicolumn{2}{|l|}{ Symptoms, no. (\%) } \\
\hline Fever & $15(33.3)$ \\
\hline Cough & 16 (35.6) \\
\hline Sore throat & $4(8.9)$ \\
\hline Shortness of breath & $6(13.3)$ \\
\hline Intensive care unit admission, no. (\%) & $2(4.4)$ \\
\hline Mothers needing intubation, no. & 0 \\
\hline \multicolumn{2}{|l|}{$\begin{array}{l}\text { Treatment with medications for COVID-19, no. } \\
(\%)\end{array}$} \\
\hline Hydroxychloroquine & $6(13.3)$ \\
\hline Azithromycin & $5(11.1)$ \\
\hline Tocilizumab & $1(2.2)$ \\
\hline \multicolumn{2}{|l|}{ Newborn characteristics } \\
\hline Birth weight, mean $( \pm S D), g$ & $3133( \pm 556)$ \\
\hline \multicolumn{2}{|l|}{ Sex, no. $(\%)$} \\
\hline Female & $19(42.2)$ \\
\hline Male & $26(47.8)$ \\
\hline Gestational age, mean (range), weeks & $34(305 / 7-41)$ \\
\hline Term ( $\geq 37$ weeks), no. (\%) & $41(91.1)$ \\
\hline Preterm (<37 weeks), no (\%) & $4(8.9)$ \\
\hline APGAR at 1 min, median & 9 \\
\hline APGAR at 5 min, median & 9 \\
\hline NICU admission, no. (\%) & $7(15.6)$ \\
\hline Prematurity & $3 / 7(42.8)$ \\
\hline Suspected sepsis & 4/7 (57.2) \\
\hline Isolation during initial practice, no. (\%) & $5(11.1)$ \\
\hline \multicolumn{2}{|l|}{ Breastfeeding and colocation, no. (\%) } \\
\hline Roomed-in & $33(73.3)$ \\
\hline Breastfeeding & $31 / 33(94)$ \\
\hline Received any breastmilk & $40(88.9)$ \\
\hline $\begin{array}{l}\text { Nasopharyngeal PCR positive for SARS-CoV-2, } \\
\text { no. }(\%)\end{array}$ & $3(6.7)$ \\
\hline Newborn with symptoms of COVID-19, no. & 0 \\
\hline \multicolumn{2}{|l|}{ Discharge disposition, no. (\%) } \\
\hline Discharge to the same household & 40/43 (93.0) \\
\hline Discharge to different household & $3(7.0)$ \\
\hline Adherence to in-person newborn visit, no. (\%) & 39/43 (90.6) \\
\hline Symptomatic on in-person newborn visit, no. & 0 \\
\hline Adherence to televisit, no. (\%) & 43/43 (100) \\
\hline Symptomatic on televisit, no. & 0 \\
\hline
\end{tabular}

Newborns were discharged to a healthy asymptomatic caregiver and were closely followed-up for 2 weeks after discharge. Televisitation was utilized in addition to initial in-person visits that showed that the newborns remained asymptomatic after discharge.

Although our study has limitations of small sample size and only short-term outcomes, we believe that to date, our report has the largest number of mother-baby dyads cared for utilizing baby friendly practices when mother is tested positive for SARS-CoV-2.

The practice of rooming-in and breastfeeding in our population provided a critical educational opportunity for the new mothers to learn isolation precautions, safe distancing, personal protective equipment use, and safe breastfeeding or pumping of breastmilk to reduce the risk of transmission of SARS-CoV-2 to the newborn. This may have prepared the SARS-CoV-2 positive mothers to better care for their newborns at home. Given the variations in the guidance and at times some conflict amongst the national and international organizations regarding colocation and breastfeeding after delivery, our experience can help hospitals when planning around best practices for newborn care during the COVID-19 pandemic [9].

Acknowledgements We would like to acknowledge and thank the medical, nursing, and support staff as well as the administration at NYC $\mathrm{H}+\mathrm{H} /$ Elmhurst Hospital for their outstanding support and extraordinary dedication while caring for the mothers and babies of Queens, NY, during the COVID-19 pandemic. We also want to thank Dr. Nitin Chouthai for his guidance and support.

\section{Compliance with ethical standards}

Conflict of interest The authors declare that they have no conflict of interest.

Publisher's note Springer Nature remains neutral with regard to jurisdictional claims in published maps and institutional affiliations.

\section{References}

1. World Health Organization. Coronavirus disease 2019 (COVID19): situation report 96-104. World Health Organization. 2020. https://www.who.int/emergencies/diseases/novel-coronavirus2019/situation-reports.

2. Coronavirus disease 2019 (COVID-19) daily data summary March 29-April 22, 2020. https://www1.nyc.gov/assets/doh/downloads/ pdf/imm/covid-19-daily-data-summary-04232020-1.pdf. 2020.

3. Puopolo K, Hudak M, Kimberlin D, Cummings J. Initial guidance: management of infants born to mothers with COVID-19. American Academy of Pediatrics Committee on Fetus and Newborn, Section on Neonatal Perinatal Medicine, and Committee on Infectious Diseases; 2020. https://services.aap.org/en/pages/2019-novel-corona virus-covid-19-infections/clinical-guidance/faqs-management-ofinfants-born-to-covid-19-mothers/. Accessed 22 April 2020.

4. Chen L, Li Q, Zheng D, Jiang H, Wei Y, Zou L, et al. Clinical characteristics of pregnant women with Covid-19 in Wuhan, China. 
N Engl J Med. 2020;382:e100. https://doi.org/10.1056/ NEJMc2009226.

5. Chen H, Guo J, Wang C, Luo F, Yu X, Zhang W, et al. Clinical characteristics and intrauterine vertical transmission potential of COVID-19 infection in nine pregnant women: a retrospective review of medical. Lancet. 2020;395:809-15. https://doi.org/10. 1016/S0140-6736(20)30360-3.

6. Chen D, Yang H, Cao Y, Cheng W, Duan T, Fan C, et al. Expert consensus for managing pregnant women and neonates born to mothers with suspected or confirmed novel coronavirus (COVID19) infection. Int J Gynaecol Obstet. 2020;149:130-6.
7. Office of the New York City Comptroller. NYC housing brief: hidden households. Office of the New York City Comptroller; 2015. https://comptroller.nyc.gov/reports/hidden-households/. Accessed 22 April 2020.

8. Centers for Disease Control. Considerations for inpatient obstetric healthcare settings. Centers for Disease Control. 2020. https://www.cdc.gov/coronavirus/2019-ncov/hcp/inpatientobstetric-healthcare-guidance.html.

9. Gupta M, Zupancic JAF, Pursley D. Caring for newborns born to mothers with COVID-19: more questions than answers. Pediatrics. 2020. https://doi.org/10.1542/peds.2020-001842. 